\title{
Digital reflection-mode time-reversed ultrasonically encoded (TRUE) optical focusing
}

Yuta Suzuki, Jian Wei Tay, Qiang Yang, Lihong Wang

Yuta Suzuki, Jian Wei Tay, Qiang Yang, Lihong Wang, "Digital reflectionmode time-reversed ultrasonically encoded (TRUE) optical focusing," Proc. SPIE 8943, Photons Plus Ultrasound: Imaging and Sensing 2014, 89431B (3 March 2014); doi: 10.1117/12.2037952

SPIE. Event: SPIE BiOS, 2014, San Francisco, California, United States 


\title{
Digital reflection-mode time-reversed ultrasonically encoded (TRUE) optical focusing
}

\author{
Yuta Suzuki*, Jian Wei Tay, Qiang Yang, and Lihong Wang* \\ Optical Imaging Laboratory, Department of Biomedical Engineering, \\ Washington University in St. Louis, St. Louis, Missouri, 63130, USA
}

\begin{abstract}
To achieve localized light delivery beyond turbid layers, TRUE optical focusing has been previously implemented by both analog and digital devices. The digital scheme offers a higher energy gain than the analog version. In many biological applications, the reflection-mode configuration, which uses backscattered light from the sample, is more suitable than the transmission-mode configuration. Although reflection-mode analog TRUE focusing has been demonstrated, its digital implementation has not been explored. Here, we report a reflection-mode digital TRUE focusing to concentrate light through a turbid layer. Further, by simply moving the ultrasound focus, we show the system's dynamic focusing capability.
\end{abstract}

Keywords: optical focusing, ultrasound modulation, optical imaging, phase conjugation, time reversal, photorefractive material.

\section{Introduction}

In biomedicine, a technique to focus light at an arbitrary location inside a turbid medium is desired. However, multiple photon scattering events hinder controlled light delivery in such media. Previously, time-reversed ultrasonically encoded (TRUE) optical focusing was demonstrated to deliver focused light inside a turbid medium [1]. In TRUE, a localized ultrasound beam spectrally modulates, or 'encodes', diffuse photons at a target region inside a sample, as shown in Fig. 1(a). Then, as shown in Fig. 1(b), by phase conjugating, or time reversing such encoded light using a phase-

(a)

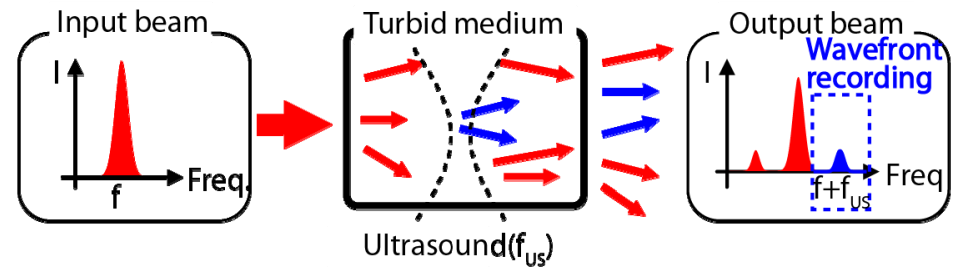

(b)

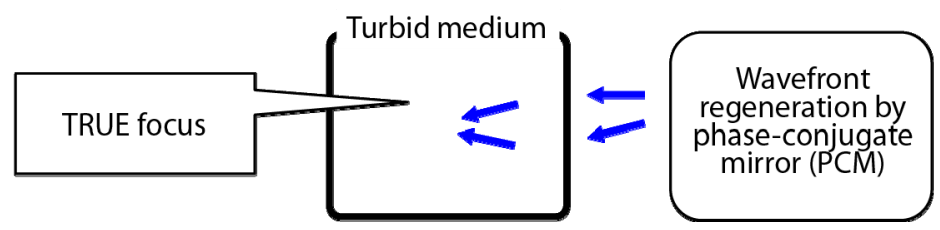

Figure 1. Principle of TRUE focusing. (a) The ultrasonic encoding of diffuse light inside a turbid medium. (b) TRUE focusing by generation of phase-conjugated light of the encoded light.

\footnotetext{
* Further author information

Yuta Suzuki, ysuzuk@wustl.edu, 1-314-935 9587

Lihong V. Wang, lhwang@,wustl.edu, 1-314- 9356152
}

Photons Plus Ultrasound: Imaging and Sensing 2014, edited by Alexander A. Oraevsky, Lihong V. Wang, Proc. of SPIE Vol. 8943, 89431B - @ 2014 SPIE · CCC code: 1605-7422/14/\$18 · doi: 10.1117/12.2037952 
conjugate mirror (PCM), light focusing at the target region is achieved. By simply moving the ultrasonic volume, light is dynamically focused at desired locations.

The implementation of PCMs were previously done by using both analog and digital devices in TRUE focusing [1-3]. In analog TRUE focusing, a photorefractive material serves as the PCM by holographically reproducing the encoded light. Because photorefractive-based PCM reproduces finer spatial details of the wavefronts than digital implementations, analog approaches have the potential to generate a higher contrast and a narrower focus than digital schemes [4]. However, due to the erasure of the hologram inside the photorefractive materials, the attainable gain, which is defined as the power ratio of the phase-conjugated light to the signal light in the recording phase, is usually limited to below 1 [2]. On the other hand, a digital PCM is realized by using a camera for wavefront recording and a spatial light modulator (SLM) for its readout. Since wavefront control by an SLM is not degraded by its readout, digital TRUE focusing has the advantage of a huge attainable energy gain, limited only by the damage threshold of the SLM if the phase conjugation is performed within the speckle correlation time of the sample. The ability to produce high gain is attractive for potential therapeutic applications of TRUE focusing. Additionally, a digital approach allows the TRUE focusing process to be repeated iteratively ('iterative focusing') [5], to improve focal qualities such as resolution, intensity, and contrast.

Although a reflection-mode analog TRUE focusing system, which phase conjugates backscattered encoded light, was previously demonstrated, the digital TRUE implementations remain in the transmission-mode configuration, which uses the light transmitted through the sample. However, it is more suitable to use the backscattered light in many biomedical applications, where accessibility to transmitted light is limited by a large attenuation of a thick tissue.

Here, we experimentally demonstrate a digital PCM in reflection mode to demonstrate TRUE focusing. Also, we show its iterative focusing abilities, which increase the TRUE focal contrast. Note that a reflection-mode setup using a single digital PCM allowed iterative focusing, while the transmission-mode configuration required two digital phaseconjugate mirrors [5]. Further, we demonstrated dynamic TRUE focusing by changing the ultrasonic-pulse positions.

\section{Experimental system}

We developed a TRUE system using a reflection-mode digital PCM, schematically illustrated in Fig. 2. The digital PCM is emphasized in the dotted block. As depicted therein, our digital PCM is in reflection-mode setup, that is, the light illumination into the sample and the diffuse-light detection are both performed on the same side of the sample. To generate a desired wavefront, we used a phase-only SLM (PLUTO, Holoeye) with a resolution of 1080×1920 pixels, which was calibrated to provide a linear phase shift over a $2 \pi$-rad range [6]. The CMOS camera imaged the conjugate plane (CP) of the SLM surface about a polarizing beam splitter (PBS). To allow one-to-one pixel matching between the CMOS camera and the SLM, an imaging lens (IL) magnified the speckle pattern at CP onto the CMOS camera.

Our digital PCM adopts the four-phase method to record the wavefront of encoded light, which was sensed by the CMOS image sensor, which was operating at a frame rate of $f_{\mathrm{CMOS}}=25 \mathrm{~Hz}$. The operation of the system consists of

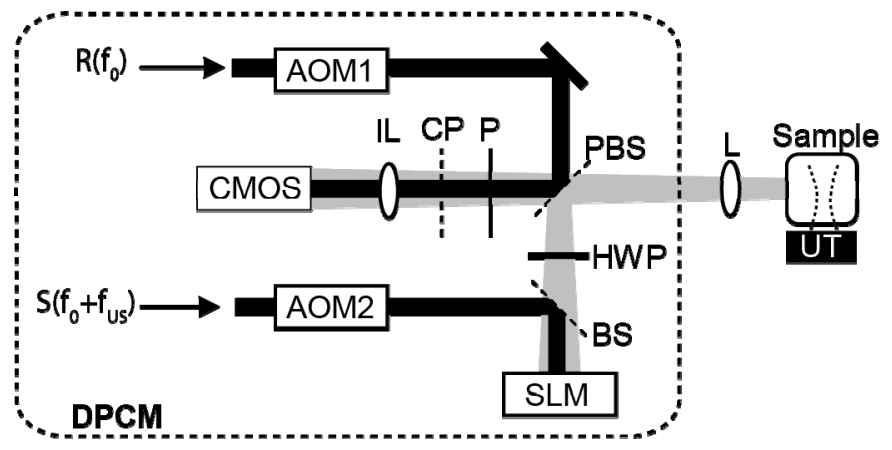

Figure 2. Optical system of TRUE focusing using reflection-mode digital phase-conjugate mirror. AOM, acousto-optic modulator; BS, beam splitter; HWP, halfwave plate; IL, imaging lens for CMOS camera; CP, conjugate plane of the SLM surface; L, lens; P, polarizer; PBS, polarizing beam splitter; R, reference beam; S, sample beam; SLM, spatial light modulator; UT, ultrasonic transducer. 
two stages. In the recording stage, a 4-cycle ultrasound pulse with the center frequency $f_{\mathrm{US}}=50 \mathrm{MHz}$ was applied to a sample. When an ultrasonic pulse was at the desired probing depth, acousto-optic modulators AOM1 and AOM2 simultaneously generated 160-ns optical pulses; one served as a reference beam $\mathrm{R}\left(f_{\mathrm{R}}\right)$, and the other as a sample beam $\mathrm{S}\left(f_{\mathrm{R}}+\Delta f\right)$, where the frequency difference was set to $\Delta f=f_{\mathrm{US}}+f_{\mathrm{CMOS}} / 4$. After being reflected by SLM, the sample beam $\mathrm{S}\left(f_{\mathrm{R}}+\Delta f\right)$ illuminated the sample in which a ultrasonic pulse spectrally modulated the frequency of diffused light. As a result of ultrasonically downshifting the $\mathrm{S}$ beam, the optical frequency becomes $f_{\mathrm{S}}-f_{\mathrm{US}}=f_{\mathrm{R}}+f_{\mathrm{CMOS}} / 4$. The down-shifted and backscattered portion $\mathrm{S}\left(f_{\mathrm{R}}+f_{\mathrm{CMOS}} / 4\right)$ was mixed with $\mathrm{R}\left(f_{\mathrm{R}}\right)$, and the CMOS image sensor captured the interferogram cycling at $f_{\mathrm{CMOS}} / 4$ for four phases. Therefore, the CMOS camera detected both the interferogram (as the intensity oscillation at $f_{\mathrm{CMOS}} / 4=6.25 \mathrm{~Hz}$ ) and the stationary background formed by other spectral components. To have a sufficient number of photons, the optical and ultrasound pulses were repeatedly fired at a repetition rate of $500 \mathrm{kHz}$ during the CMOS camera exposure time of $35 \mathrm{~ms}$. Since the frequency of the intensity oscillation was at $f_{\mathrm{CMOS}} / 4$, four CMOS frames corresponded to four phases of the interferogram. From 64 CMOS images of an interferogram, which corresponded to 16 cycles of the intensity oscillation, we obtained the wavefront of the encoded light by calculating the phases of the oscillation at each pixel. Together, to evaluate the system operation, we measured the SNR of the interferogram by first calculating the power ratio of the intensity oscillation to the noise for each CMOS pixel, and then averaging the overall pixel values.

In the readout phase, the measured wavefront was displayed on the SLM, after being corrected for the premeasured SLM curvature, which was measured using a Michelson interferometer prior to the experiment. After closing the shutter in front of the CMOS sensor to prevent saturation, the readout was performed using a continuouswave readout beam, which was generated by turning on AOM2. Note that the wavefront readout produces not only a TRUE focus but also diffuse background light due to an incomplete phase conjugation, which can be digitally subtracted by adaptive background subtraction [2].

\section{Focus visualization}

To demonstrate the system's focusing capability, we visualized the TRUE focus by generating it on a fluorescent quantum dot sheet placed behind a turbid layer with a thickness of 0.7 transport mean free path $\left(l_{t}^{\prime}\right)$ and a reduced scattering coefficient of $100 \mathrm{~cm}^{-1}$, whose experimental configuration is shown in Fig. 3(a). A lens with a $60 \mathrm{~mm}$ focal length focused light onto the sample. The excited fluorescent light on the sheet was imaged by a CCD camera placed above the sample. A long-pass filter was used to select fluorescent light at $600 \mathrm{~nm}$, and to reject the light at an excitation wavelength of $532 \mathrm{~nm}$. To generate the TRUE focus, we focused the ultrasound pulse on the fluorescent sheet and adjusted the time delay between the optical and ultrasound pulses so that the optical pulses illuminated the sample when the ultrasound pulse was on the fluorescent sheet. An aluminum reflector was placed behind the turbid layer to increase the backscattered light intensity.

When the SLM was set to display a uniform pattern, although the turbid layer was thinner than $1 l_{t}^{\prime}$, it effectively scrambled the light propagation, and we observed the diffused fluorescent excitation on the fluorescent sheet, as shown in Fig. 3(b). As an initial TRUE focusing experiment, we used a uniform SLM pattern for sample illumination to capture the wavefront of the ultrasonically encoded light. Then, we read out the phase-conjugated light of the encoded light by displaying the captured wavefront on the SLM to generate a TRUE focus (single-shot TRUE focusing). To observe the TRUE focus, we captured two excited fluorescent CCD images on the fluorescent sheet, one excited by the phaseconjugate wavefront, and the other excited by the unfocused diffuse background, and the two images were subtracted. To generate the background excitation, we formed a background phase map from the recorded wavefront by alternatively adding 0 and $\pi$ rad to $5 \times 5$ blocks that spanned the SLM. Fig. 3(c) shows the distribution of the excited fluorescent light by single-shot TRUE focusing, and Fig. 3(d) is the unfocused background of the TRUE focus. We subtracted the image in Fig. 3(d) from Fig. 3(c) to see the TRUE focus more clearly, and the background-subtracted image is shown in Fig. 3(e). However, as shown, we did not observe any noticeable TRUE focus, because of the weak signal-to-noise ratio of the wavefront recording of the encoded light.

To increase the contrast of TRUE focusing, we performed the wavefront recording and readout of the encoded light by repeatedly illuminating the sample by TRUE focusing to record another hologram (referred to hereafter as 'iterative focusing'). Such an approach was previously demonstrated to increase the contrast of TRUE focus in transmission-mode setup [5]. In contrast to the transmission-mode setup, our reflection-mode setup allowed us to 
iteratively focus using a single digital PCM. After 20 such iterations, the intensity of the TRUE focus increased, as shown in Fig. 3(f). The fluorescent excitation caused by an unfocused background is shown in Fig. 3(g). The image after subtracting the background is shown in Fig. 3(h), where we see the improved contrast of the TRUE focus over singleshot TRUE focusing, and we observed a clear focused line on the QD fluorescent sheet.

The observed contrast of the TRUE focus is currently weak, however, probably due both to the low signal-tonoise ratio of recording encoded light wavefronts, and to imperfect pixel matching between the CMOS sensor and SLM. However, we expect that we can improve the contrast by better pixel matching. We can also improve the contrast by increasing the signal-to-noise ratio with the aid of advanced filtering techniques such as spectral hole burning and photorefractive interferometers.

(a)

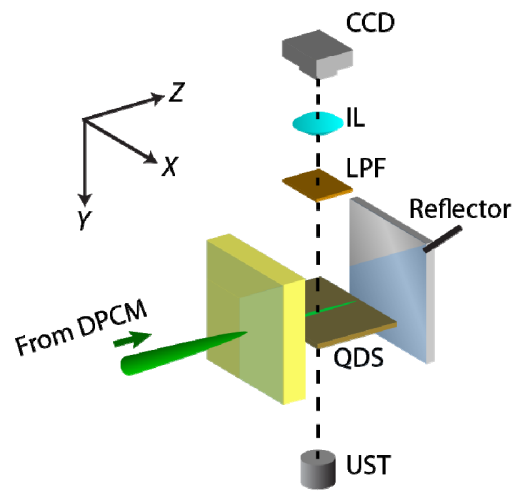

(c)

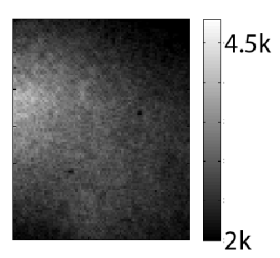

(f)

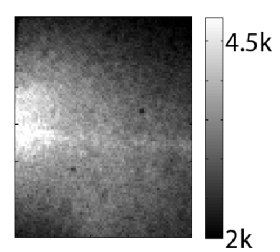

(d)

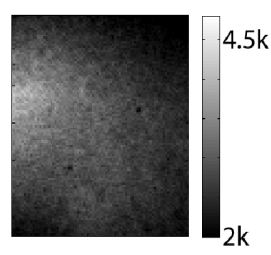

(g)

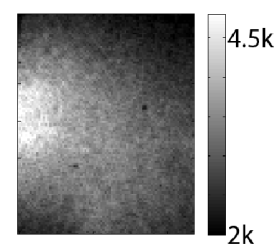

(b)

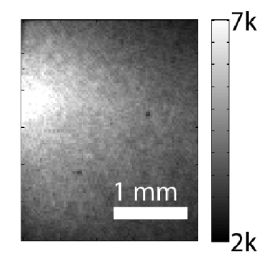

(e)

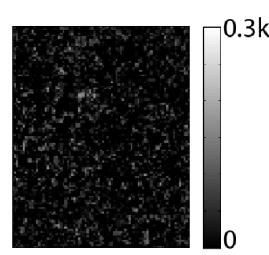

(h)

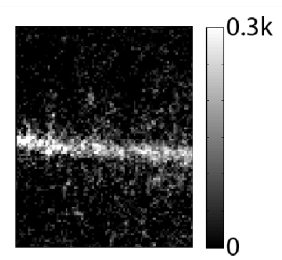

Figure 3. Contrast improvement of TRUE focus by iterative focusing using reflection-mode digital PCM. (a) Experimental configuration. CCD, charge coupled device; IL, imaging lens; LPF, long pass filter; QDS, quantum dot sheet; UST, ultrasonic transducer; DPCM, digital phase-conjugate mirror. (b) Fluorescent excitation by displaying an uniform phase pattern on SLM. (c) Excited fluorescent excitation by single-shot TRUE focusing. (d) Diffuse background excitation of single-shot TRUE focusing. (e) After background subtraction from (c). (f) Excited fluorescent excitation by iterative focusing ( $\mathrm{n}=20)$. (g) Diffuse background excitation of iterative focusing by shifting 10 pixel. (h) Excited fluorescence by TRUE focusing after background subtraction.

\section{Dynamic TRUE focusing}

To demonstrate the dynamic TRUE focal positioning using our system, we captured TRUE fluorescent excitation images on a QD sheet at different ultrasound positions, using the CCD camera mounted above the sample. The sample schematic was the same as that in Fig. 3(a), and the CCD imaged the 2-D fluorescent distributions on a QD sheet placed behind a turbid layer of $0.5 l_{\mathrm{t}}^{\prime}$ with a reduced scattering coefficient $\mu_{\mathrm{s}}{ }^{\prime}=0.25 \mathrm{~cm}^{-1}$. After performing 5 iterations of the TRUE focusing process, we captured the excited fluorescent distribution on the fluorescent sheet by TRUE focusing during the readout stage. Also, the fluorescent excitations caused by the unfocused surrounding background light were measured in the same manner as before and subtracted from the excitation during the readout stage, to increase the 
observed contrast of TRUE focus. To demonstrate the dynamic focusing ability of our system, we translated the ultrasonic transducer and obtained the fluorescence distribution excited by TRUE focus at each ultrasonic position. The step size of the scan was set to $\Delta x=0.13 \mathrm{~mm}$.

As a result of scanning the ultrasound position, the observed fluorescent excitation by the TRUE focus was translated according to the ultrasonic positional shift, as shown in Fig. 4 (a)-(h). The scanning range in this experiment was limited by a signal-to-noise ratio of the wavefront sensing, which may limit our field of view when we image through a turbid medium. However, an improvement in the scanning range is expected by increasing the number of iterations because this would increase the amount of encoded light over each iteration, and hence also increase the signal-to-noise ratio.

(a)

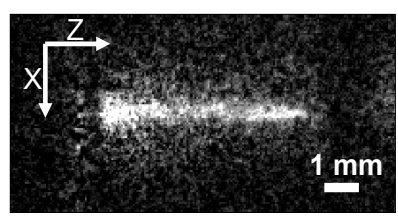

(e)

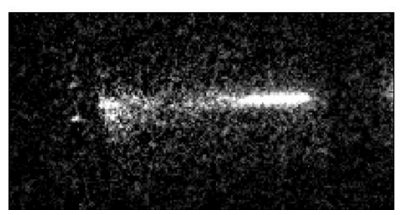

(b)

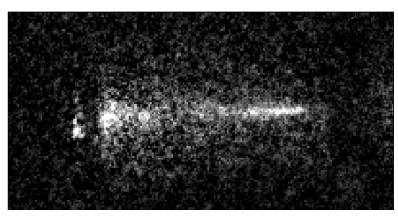

(f)

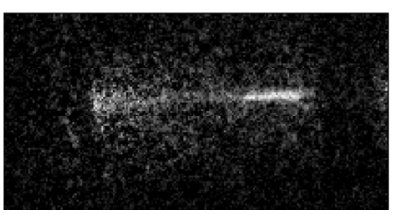

(c)

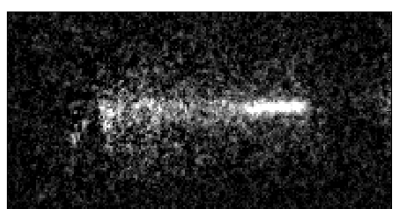

(g)

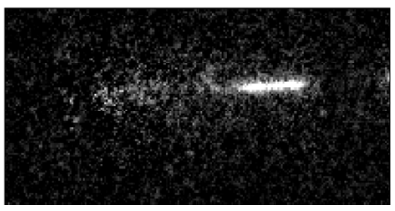

(d)

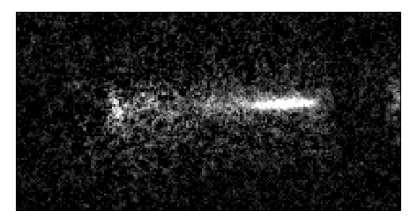

(h)

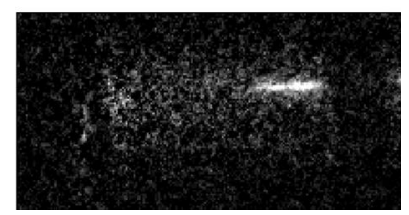

Figure 4. Dynamic TRUE focal positioning via shifting ultrasound position at an interval of $0.13 \mathrm{~mm}$ along $x$ axis. (a)-(h) Fluorescent excitation by TRUE focusing when $x$ position of ultrasound transducer are $0 \mathrm{~mm}, 0.13 \mathrm{~mm}, 0.26 \mathrm{~mm}, 0.39 \mathrm{~mm}, 0.52$ $\mathrm{mm}, 0.65 \mathrm{~mm}, 0.78 \mathrm{~mm}$, and $0.91 \mathrm{~mm}$, respectively. The unfocused background are digitally subtracted from the phaseconjugated readout excitation images.

\section{Conclusions}

In summary, we have demonstrated reflection-mode digital TRUE focusing through a turbid medium, along with iterative focusing. The TRUE focusing capability through a turbid layer was verified by visualizing fluorescent excitation by TRUE focus through a turbid layer. Although single-shot TRUE focusing did not produce a noticeable TRUE focus, through iterative focusing, we managed to observe the focused beam through a turbid layer. Although currently, the use of a reflector behind the turbid layer is necessary due to the low amount of encoded light signal, potentially we can increase the signal-to-noise ratio by advanced filtering techniques, such as spectral hole burning and photorefractive interferometers [7-9]. Further, by merely translating the positions of ultrasound volume, we were able to translate the TRUE focus accordingly.

\section{Acknowledgements}

This work was sponsored in part by National Institutes of Health grants DP1 EB016986 (NIH Director's Pioneer Award) and R01 CA186567 (NIH Director's Transformative Research Award). L.W. has a financial interest in Microphotoacoustics, Inc. and Endra, Inc., which, however, did not support this work.

\section{References}

[1] Xu, X., Liu, H. L., and Wang, L. V., "Time-reversed ultrasonically encoded optical focusing into scattering media," Nature Photonics, 5, 154-157 (2011). 
[2] Wang, Y. M., Judkewitz, B., DiMarzio, C. A., and Yang, C. H., "Deep-tissue focal fluorescence imaging with digitally time-reversed ultrasound-encoded light," Nature Communications, 3, 928-935 (2012).

[3] Si, K., Fiolka, R., and Cui, M., "Fluorescence imaging beyond the ballistic regime by ultrasound-pulse-guided digital phase conjugation," Nature Photonics, 6, 657-661 (2012).

[4] Lai, P., Suzuki,Y., Xu, X. and Wang, L. V., "Focused fluorescence excitation with time-reversed ultrasonically encoded light and imaging in thick scattering media," Laser Physics Letters, 10, 075604 (2013).

[5] Si, K., Fiolka, R., and Cui, M., "Breaking the spatial resolution barrier via iterative sound-light interaction in deep tissue microscopy," Scientific reports, 2, 748-751 (2012).

[6] Tay, J. W., Taylor, M. A., and Bowen, W. P., "Sagnac-interferometer-based characterization of spatial light modulators," Applied Optics, 48, 2236-2242 (2009).

[7] Li, Y., Hemmer, P. L., Kim, C., Zhang, H., and Wang, L. V., "Detection of ultrasound-modulated diffuse photons using spectral-hole burning," Optics Express, 16, 14862 (2008).

[8] Murray, T. W., Sui, L., Maguluri, G., Roy, R. A., Nieva, A., Blonigen, F. J., and DiMarizo, C. A., "Detection of ultrasound-modulated photons in diffuse media using the photorefractive effect," Optics Letters, 29, 2509-2511 (2004). [9] Rousseau, G., Blouin, A., and Monchalin, J.-P., "Ultrasound-modulated optical imaging using a high-power pulsed laser and a double-pass confocal Fabry-Perot interferometer," Optics Letters, 34, 3445-3447 (2009). 